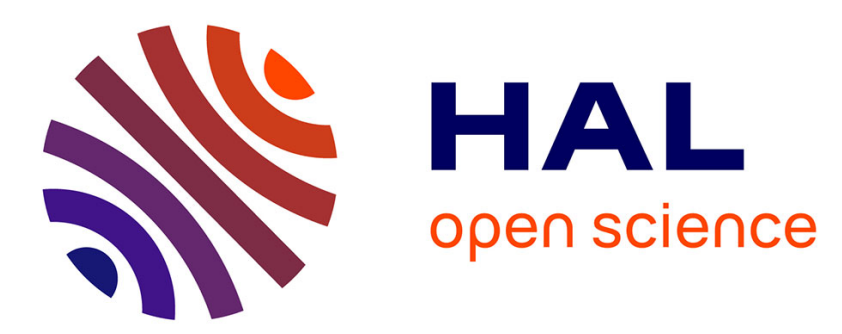

\title{
Morphological tools for spatial and multiscale analysis of passive microwave remote sensing data
}

Sébastien Lefèvre, Erchan Aptoula

\section{To cite this version:}

Sébastien Lefèvre, Erchan Aptoula. Morphological tools for spatial and multiscale analysis of passive microwave remote sensing data. Specialist Meeting on Microwave Radiometry and Remote Sensing of the Environment (MicroRad), 2016, Espoo, Finland. hal-01253875

\section{HAL Id: hal-01253875 \\ https://hal.science/hal-01253875}

Submitted on 13 Nov 2019

HAL is a multi-disciplinary open access archive for the deposit and dissemination of scientific research documents, whether they are published or not. The documents may come from teaching and research institutions in France or abroad, or from public or private research centers.
L'archive ouverte pluridisciplinaire HAL, est destinée au dépôt et à la diffusion de documents scientifiques de niveau recherche, publiés ou non, émanant des établissements d'enseignement et de recherche français ou étrangers, des laboratoires publics ou privés. 


\section{MORPHOLOGICAL TOOLS FOR SPATIAL AND MULTISCALE ANALYSIS OF PASSIVE MICROWAVE REMOTE SENSING DATA}

\author{
S. Lefèvre* \\ Université Bretagne Sud \\ UMR 6074 IRISA \\ Vannes, France
}

E. Aptoula ${ }^{\dagger}$

\author{
Gebze Technical University \\ Institute of Information Technologies \\ Kocaeli, Turkey
}

\begin{abstract}
Earth Observation through microwave radiometry is particularly useful for various applications, e.g., soil moisture, ocean salinity, or sea ice cover. However, most of the image processing/data analysis techniques aiming to provide automatic measurement from remote sensing data do not rely on any spatial information, similarly to the early years of opti$\mathrm{cal} / \mathrm{hyp}$ erspectral remote sensing. After more than a decade of research, it has been observed that spatial information can very significantly improve the accuracy of land use/land cover maps. In this context, the goal of this paper is to propose a few insights on how spatial information can benefit to (passive) microwave remote sensing. To do so, we focus here on mathematical morphology and provide some illustrative examples where morphological operators can improve the processing and analysis of microwave radiometric information. Such tools had great influence on multispectral/hyperspectral remote sensing in the past, and are expected to have a similar impact in the microwave field in the future, with the launch of upcoming missions with improved spatial resolution, e.g. SMOS-NEXT.

Index Terms - Mathematical Morphology, Multiscale representation, Feature extraction, Spatial information, Remote sensing
\end{abstract}

\section{INTRODUCTION}

Earth Observation benefits from a wide range of satellite sensors. Among the available imaging modalities, microwave radiometry is particularly useful for various applications, e.g., soil moisture, ocean salinity, or sea ice cover. Such applications have gathered numerous works aiming to provide automatic measurements from analysis of remote sensing data. However, most of these image processing/data analysis techniques do not rely on any spatial information, similarly to the early years of optical/hyperspectral remote sensing. After

\footnotetext{
*Thanks to French National Research Agency (ANR) - Project Grant ANR-13-JS02-0005-01 (Asterix project) for funding.

${ }^{\dagger}$ Thanks to Scientific and Technological Research Council of Turkey (TUBITAK) - Career Grant 112E210 for funding.
}

more than a decade of research, it has been observed that spatial information can very significantly improve the accuracy of land use/land cover maps. The object-based image analysis (OBIA) paradigm, computation of multiscale features, spatial regularization of classification maps are among the various techniques that have shown great success for remote sensing of the environment. Surprisingly, such advances have not impacted the field of passive microwave remote sensing yet. Meanwhile, the launch of new missions, e.g., ESA SMOS (2009), NASA/JAXA GMP (2014), or NASA SMAP (2015), brings new data that call for the development of new efficient and accurate image processing algorithms.

In this context, the goal of this paper is to propose a few insights on how spatial information can benefit to (passive) microwave remote sensing. To do so, we focus on Mathematical Morphology (MM), a nonlinear image analysis framework equipped with rigorous mathematical foundations and efficient tools for exploiting spatial information. Remote Sensing has been a successful application field of MM [1], with developments such as local descriptors for land cover classification, global descriptors for (patch) image retrieval, object detection based on shape/geometric properties, or (multiscale) image segmentation. We inspired from these works and provide here three different examples where morphological operators can improve the processing and analysis of microwave radiometric information: (i) image simplification with quasi-flat zones filtering, (ii) peak detection with top-hat by reconstruction, and (iii) multiscale description for temperature classification. All experiments are conducted on a standard SMOS dataset.

The paper is organized as follows. In Sec. 2, we provide the general reader some introduction to mathematical morphology. We then describe the three illustrative applications in Sec. 3, before discussing open challenges in Sec. 4 and providing concluding remarks in Sec. 5 .

\section{MORPHOLOGICAL TOOLS}

Mathematical Morphology has been introduced in the 1960s by Serra and Matheron in the context of material sciences. Since then, it has become a popular toolbox in image pro- 
cessing [2], with sucessful applications in many contexts including remote sensing [1]. While initially defined on binary images using the set theory, it has been later extended to the general case of greyscale images using the lattice theory.

\subsection{Structuring Operators}

Mathematical Morphology nowadays provides a wide range of tools. For the sake of illustration, we recall here only the most basic ones, which aim to probe an image $f$ with a local template $b$ (also called structuring element).

The two elementary operations are called erosion $\varepsilon$ and dilation $\delta$. They respectively shrink and enlarge objects present in the image (or compute a local minimum or maximum):

$$
\begin{aligned}
& \varepsilon_{b}(f)(p)=\bigwedge_{q \in b} f(p+q), p \in E \\
& \delta_{b}(f)(p)=\bigvee_{q \in \breve{b}} f(p+q), \quad p \in E
\end{aligned}
$$

where $\breve{b}=-b$ is the symmetric template of $b$. These operators can be further combined to build morphological filters such as opening and closing that respectively acts in removing all foreground and background components of the image in which the template does not fit:

$$
\begin{aligned}
\gamma_{b}(f) & =\delta_{\breve{b}}\left(\varepsilon_{b}(f)\right) \\
\varphi_{b}(f) & =\varepsilon_{\breve{b}}\left(\delta_{b}(f)\right)
\end{aligned}
$$

Let us note that many other operators can be derived from these basic ones [2]. The advantages of MM is the rigorous mathematical framework, with morphological operators being translation-invariant, increasing, and for opening/closing being also anti-extensive/extensive and idempotent. Furthermore, efficient algorithms and implementations of these operators have been introduced in the literature, and many morphological image analysis toolboxes are available.

\subsection{Connected and Hierarchical Approaches}

The major drawback of previous morphological operators is the need for a structuring element, whose shape and size have a great impact on the final result. Connected operators were thus introduced to solve this issue. Furthermore, these filters have also a tremendous advantage, since they do not change nor add image contours but only filter out some objects by merging elementary regions called flat zones. To illustrate, instead of filtering out the elements that do not contain a $5 \times 5$ square, it is possible to remove those that do not contain at least 25 pixels. For more details on connected approaches, the reader is refer to the tutorial [3].

Connected operators can be built following two distinct approaches. The first one consists in filtering the image by applying a structural operator, before reconstructing image contours using a geodesic reconstruction process. It leads to the so-called filters by reconstruction. The second strategy requires to first build a hierarchical representations of the image, known as a (morphological) tree. Various tree models are available: min- and max-tree, tree of shapes, binary partition tree, $\alpha$-tree, etc. Selected nodes and branches are then filtered out using a tree pruning process. The resulting image can finally be reconstructed from the simplified tree. This strategy is particularly appealing since processing a tree structure is much more efficient than a raster analysis requiring to scan all pixels.

\section{APPLICATIONS}

Let us recall that our objective is to assess the interest of MM in microwave remote sensing. To do so, we will illustrate the potential of morphological operators on a standard dataset, namely the Murrumbidgee River catchment [4]. Our experiments were conducted using the level $1 \mathrm{C}$ product (MIR_SCLF1C), from the observation acquired on 29/03/2016 (20:18:27 - 21:11:45). In this preliminary work, we are using only the brightness temperature information. For the sake of simplicity, we have use digital images defined on a standard square grid, using the Earth fixed grid point system. We have not tackled complex data and thus we only rely on $\mathrm{H} \& \mathrm{~V}$ polarization channels. Let us note that we use here polarization computed at the surface level frame and not at the antenna frame.

\subsection{Image Simplification with Quasi-Flat Zones Filtering}

The first application aims to simplify the content of a SMOS image, with an edge-preserving image simplification technique. Contrary to common linear filtering techniques such as Gaussian smoothing, connected morphological filtering allows removing image details while keeping the original location of image contours. To do so, we model the image through quasi-flat zones (QFZ), that offer a robust extension of flat zones (FZ) or connected components (CC). A FZ is made of all neighboring pixels that share exactly the same image value. In many cases, the pixels belonging to the same object have very similar (but not strictly equal) values, thus rendering the concept of FZ useless when dealing with integer- or real-valued images. By extension, a QFZ allows some variation of the image values.

Two main definitions of QFZ have been introduced in the literature [5]. The $\alpha$-CC gathers pixels such as, for any couple of pixels in the QFZ, there exists a path linking these two pixels with a local difference not higher than $\alpha$ between two neighboring pixels along the path. This model only relies on local differences that are compared with a local range threshold. Its main drawback is its sensitivity to blurred edges, that lead to the chaining effect. To explain, let us consider a shading from 0 to 100 , with a local difference of 1 between two neighboring pixels. All the pixels will then belong to the 
same, unique, $\alpha$-CC if $\alpha=1$. Setting $\alpha=0$ produces $\mathrm{FZ}$ or standard CC, and here every single pixel will lead to a distinct component.

An extension of the $\alpha$-CC has been introduced by Soille in [5]. Instead of using only a local range threshold, it is possible to also take into account the global range of a QFZ. It leads to the concept of $(\alpha, \omega)$-CC, where the maximal difference between any pair of pixels belonging to the same QFZ has to be lower than a threshold $\omega$. In practice, it has been shown that both local $(\alpha)$ and global $(\omega)$ thresholds can be conveniently assigned the same value. A higher $\alpha=\omega$ value means that higher differences between pixels of the same QFZ are allowed. In other words, the QFZ are made of a more heterogeneous content, and there are less QFZ in the image. The filtering effect is thus stronger.

We show the behavior of this filtering on SMOS data in Fig. 1, where visual illustrations are given. We consider both $\mathrm{H}$ and $\mathrm{V}$ polarizations, on the left and right column respectively. The first line shows original images, with their contrast being stretched for the sake of clarity in second line (for the $\mathrm{V}$ polarization, contrast stretch is applied in the $[5 \%-95 \%]$ range). Then successive filtering results are reported, with increasing $\alpha=\omega$ values, from 3 to 30 (assuming here a byte representation of pixel values, i.e. pixels taking values in $[0,255])$. We can visually observe the filtering effect, with less and less remaining objects, but always keeping the actual borders of image components. Let us note that the filtering was always applied on the initial images, and contraststretched versions are provided here only to ease understanding.

Beyond visual assessment, we also provide a quantitative evaluation in Tab. 1. While the initial $\mathrm{H}$ and $\mathrm{V}$ images contain 57876 pixels, modeling the image through its connected components allows reducing the number of elements to 1930 flat zones (i.e., with $\alpha=\omega=0$ ). These flat zones actually correspond to individual cells discretized on the square grid. Furthermore, when considering QFZ instead of FZ, the number of elements decreases significantly. It is interesting to notice that for low values of $\alpha, \omega$, the image complexity (measured in terms of number of elements) is reduced by a factor of 3, while the visual appearance of images is kept.

The proposed morphological filtering offers two main advantages. On the one hand, it consists in an efficient way to perform spatial regularization, without requiring to define a fixed neighborhood conversely to most of the other regularization techniques (e.g. Markov Random Fields). Indeed, the neighborhood of a pixel actually depends on the image content, and is extended up to the $(\alpha, \omega)$ range. On the other hand, such a simplification into flat or quasi-flat zones allows a strong reduction of the number of image elements. This kind of data compression is appealing since it leads to faster subsequent processing (e.g. data assimilation of the brightness temperature values).
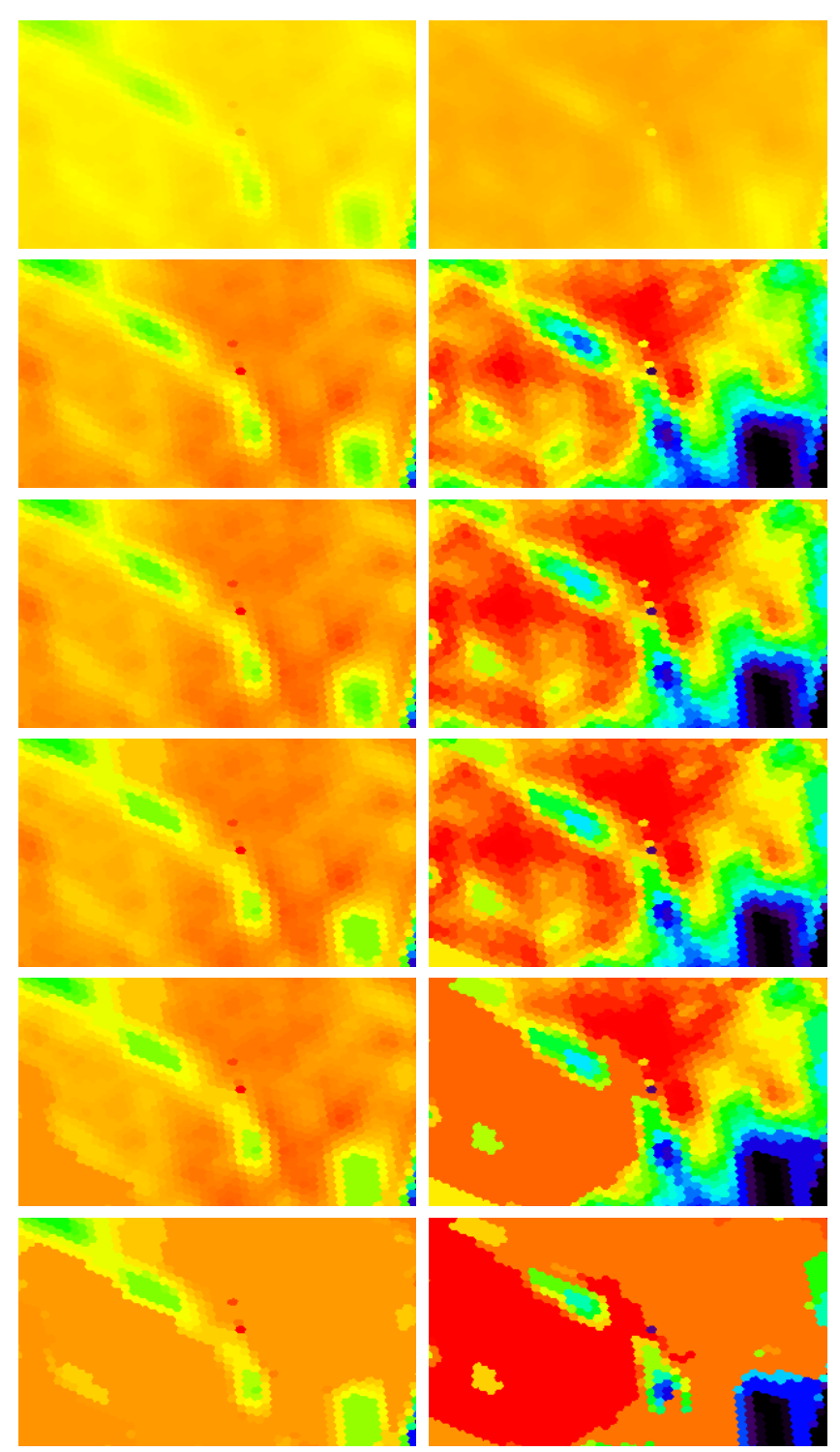

Fig. 1. Image simplification on the $\mathrm{H}$ (left) and V (right) polarization data: (from top to bottom) original images and their contrast-stretched versions, filtered images using increasing $\alpha, \omega$ values: $3,9,18$, and 30 .

Table 1. Quantitative assessment of image simplification.

\begin{tabular}{lcccccc}
\hline Polarization & \multicolumn{2}{c}{ Unfiltered } & \multicolumn{4}{c}{ QFZ, with $\alpha=\omega$ set to } \\
& Pixels & FZ & 3 & 9 & 18 & 30 \\
\hline $\mathrm{H}$ & 57876 & 1930 & 677 & 590 & 534 & 156 \\
$\mathrm{~V}$ & 57876 & 1930 & 602 & 562 & 365 & 96 \\
\hline
\end{tabular}




\subsection{Peak Detection with Top-Hat by Reconstruction}

The second application of morphological tools deals with peak detection. While opening (resp. closing) aims to filter the image through removal of its small foreground (resp. background) image elements, it is also possible to focus on these removed elements. The resulting operators are called residue operators, and basically compare a filtered image with its original version. Morphological top-hat is the most common residue operator, and is defined as the difference between the original image and the result of an opening (white top-hat) or a closing (black top-hat, also called bottom-hat). While the white top-hat returns small objects (i.e. that fit within a structuring element) brighter than their surroundings, the black top-hat returns objects darker than their surroundings. Furthermore, to avoid the artifacts brought by the shape of the structuring element, these residue operators may be favorably replaced by their connected version. To do so, opening and closing are respectively replaced by opening and closing by reconstruction (thus keeping image contours).

We apply top-hat by reconstruction on both $\mathrm{H}$ and $\mathrm{V}$ polarization data, and report results in Fig. 2 (left and right columns, respectively). We consider two different sizes for the structuring element. In the first case (middle row), we extract objects made of a single element (hexagonal cell) brighter than their surrounding. The corresponding structuring element has been empirically defined as a $7 \times 7$ square. The top-hat operator provides two kinds of information, i.e. the location of residual objects (non-zero elements) as well
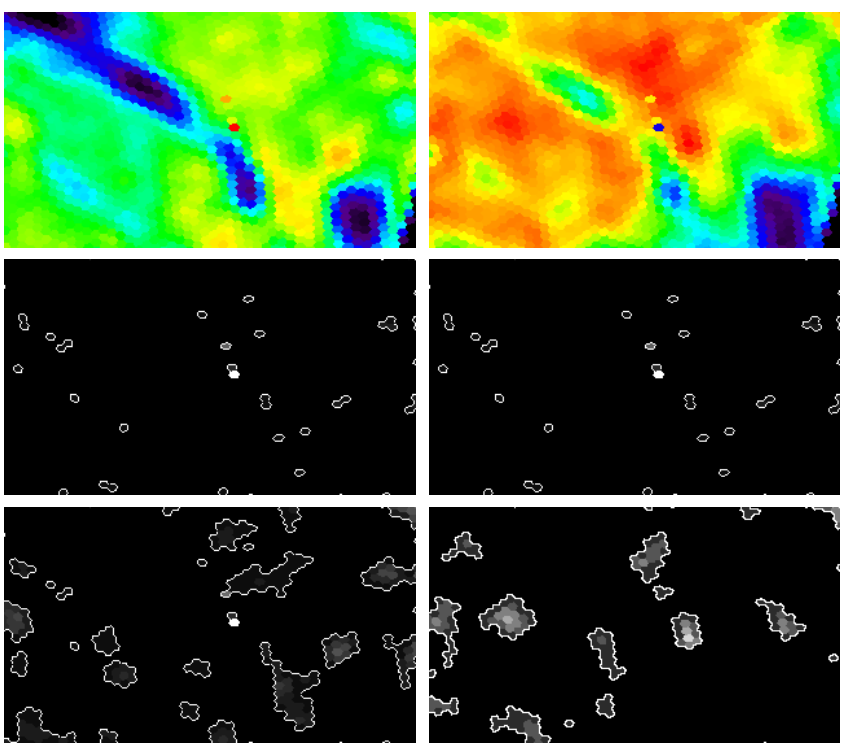

Fig. 2. Peak detection using top-hat on the $H$ (left) and $\mathrm{V}$ (right) polarization data. Top: original images (contrast stretched). Middle: top-hat of size 1 unit $(7 \times 7$ pixels). Bottom: top-hat of size 6 units $(21 \times 21$ pixels $)$. Borders of all non-zero elements have been highlighted. as their strength (measured as the difference between the original and filtered images). In Fig. 2, contours of nonzero elements are overlayed to allow identification of weak residues that would otherwise appear as very dark pixels. The resulting image contains all local image maxima. In the second case (bottom row), we considering a filtering size of 7 elements (i.e. one hexagonal cell and its 6 neighbors). The structuring element has thus been empirically set to a square of $21 \times 21$ pixels. The operator is then able to extract local maxima of larger extent. Let us note that such elements would have been very hard to extract using a thresholding technique. Besides, the only parameter required by the operator is the size of the filter. Robustness to changes of illumination and contrast is also an attractive property.

The proposed application is useful to extract small compact hot spots disconnected from hot areas. Such regions may correspond to radio frequency interferences (RFI), whose detection is an important problem in microwave remote sensing. Besides, the same principle can be applied with cold spots using the bottom hat.

\subsection{Multiscale Description and Temperature Classifica- tion}

The last experiment reported in this paper illustrates how MM can be used to improve pixel-based classification. In optical remote sensing, differential morphological profiles (DMP) [6] have been assessed as powerful multiscale descriptors in the context of (urban) land cover classification. Such profiles are built from a scale-space analysis. In other words, the input image is iteratively processed with morphological filters (most often with opening and closing operators) of increasing size. Each pixel is then described by its morphological profile, that is made of the values taken in the different filtered images. The differential morphological profile is nothing but the first derivative of this profile, storing the differences between two successive filtered images instead of the filtered images themselves. Connected operators (opening and closing by reconstruction) are used to avoid the artificial introduction of edges due to the shape of the structuring element. After a decade of intensive use, DMP have been successfully replaced by DAP, or differential attribute profiles [7]. Basically, the overall scheme is kept but the opening/closing by reconstruction are replaced by attribute opening/closing (e.g. with a size attribute). DAP features allow for both a higher flexibility (a wide range of attributes can be used) and a lower computational cost, since they can be efficiently computed from a tree-based representation.

We describe here each pixel by its DAP, thus allowing a modeling of its brightness temperature (or rather its evolution) at multiple scales. Each scale corresponds to an attribute filtering, that is achieved through max-tree pruning. We use the standard area attribute, measured in number of pixels. Since we do not have any ground truth or reference 
data (provided as a reference map with predefined classes), we consider here an unsupervised classification setup. We use the popular K-means clustering technique, and split the feature space (i.e. original $\mathrm{H}$ and $\mathrm{V}$ values, or their multiscale counterparts) into three classes. Fig. 3 provides classification maps that are produced from the original $\mathrm{H}$ and $\mathrm{V}$ images (recalled in (a) and (b)) by the K-means algorithm, when using the initial brightness temperature data from $\mathrm{H}(\mathrm{c}), \mathrm{V}(\mathrm{d})$, or both $\mathrm{H}$ and $\mathrm{V}(\mathrm{g})$. DAP are computed from these two polarization images, and clustering is applied on DAP features either computed from $\mathrm{H}$ only (e), V only (f), or from both $\mathrm{H}$ and $\mathrm{V}(\mathrm{h})$.

Without any ground truth available, it is difficult to assess the accuracy of any of these classification maps. But the different maps provided in Fig. 3 show that clustering based on DAP information clearly differs from the baseline using original temperature brightness values at the standard scale. We can visually observe that DAP allows taking spatial informa-

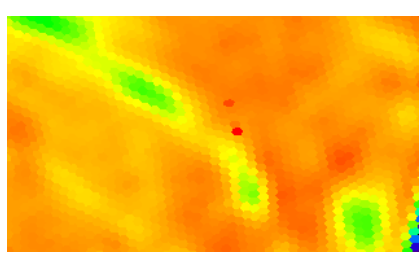

(a)

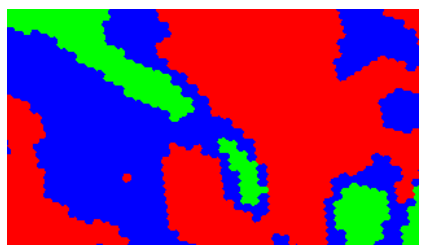

(c)

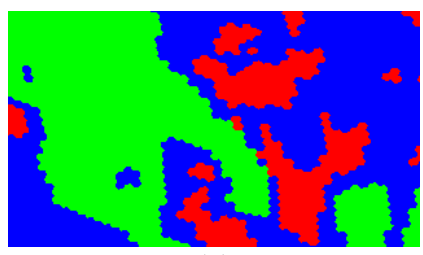

(e)

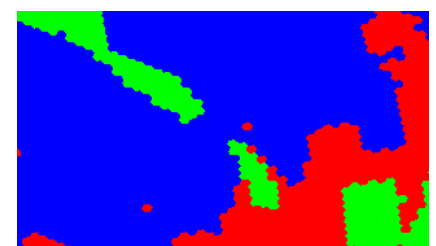

(g)

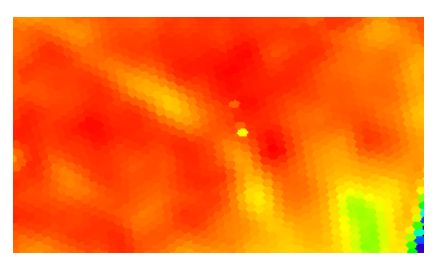

(b)

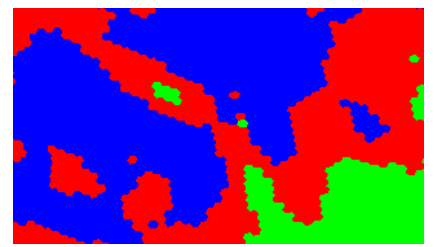

(d)

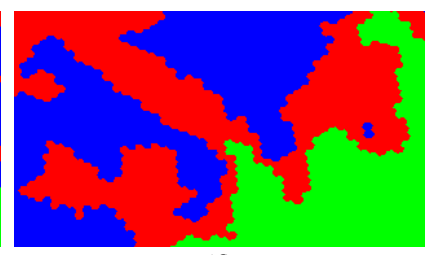

(f)

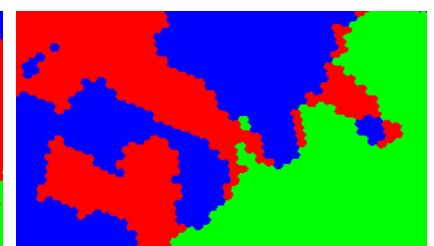

(h)
Fig. 3. Image clustering using DAP from brightness temperature measurements. From top to bottom: original (contraststretched) $\mathrm{H}$ (a) and V (b) polarization data, clustering using only brightness temperature values from $\mathrm{H}(\mathrm{c})$ and $\mathrm{V}(\mathrm{d})$, using DAP from $\mathrm{H}$ (e) and V (f), and using brightness temperature $(\mathrm{g})$ or DAP $(\mathrm{h})$ from both $\mathrm{H}$ and $\mathrm{V}$. tion into account. Nevertheless, some supervised classification settings should be considered for quantitative assessment.

To summarize, this experiment shows that MM makes possible the characterization of both spatial and spectral information. DAP description offers a higher discriminating power but at the cost of longer feature vectors (i.e. the number of scales). Let us observe that we have here only relied on the area attribute due to its popularity, but nothing prevents us using other attributes.

\section{DISCUSSION}

The different experiments described in the previous section illustrate how morphological approaches could contribute to the analysis of microwave remote sensing data. From these first attempts, we have identified several challenges to be addressed for a complete application of morphological operators.

The SMOS instrument provides multi-angular information, where brightness temperature are observed from multiple incidence angles. In this paper, we have reported preliminary experiments using the so-called browse product where a unique observation is built from the multi-angular observations. Undoubtedly, processing the full multi-angular information is expected to provide better results. However, this requires defining morphological tools able to cope with such multi-angular data. Such a requirement calls for new developments in the field of MM.

Furthermore, the SMOS instrument is monitored in full polarization mode. It means that X, XY, Y, XY polarizations are recorded alternatively. We have used here only $\mathrm{X}$ and $\mathrm{Y}$ polarizations, and more precisely their mapped values $\mathrm{H}$ and $\mathrm{V}$ at the surface level frame. A deeper analysis of the data would mean to process XY polarizations too, that come in a complex numeric representation. Defining morphological operators on complex-valued data has led to only a few studies, and needs to be further explored in the context of passive microwave remote sensing data.

Another challenge is related to the spatial grid considered for the analysis. In SMOS products, data are sampled along a non-regular grid, using the Icosahedral Snyder Equal Area projection (ISEA4H9). Such a grid differs from the standard square grid commonly used in digital image processing. In order to exploit as best as possible the specificity of SMOS products, new connectivity measures have to be used for hexagonal cells to be able to identify the neighbors of each image element. Since MM relies on a spatial analysis of image content, such a definition has a very strong influence on the final result.

While brightness temperature values from a L1C product were considered here, let us observe that morphological tools can be applied to other microwave-related data, e.g. Soil Moisture or Ocean Salinity maps. In this context, the goal could be to achieve a spatial post-processing of the SM or OS 
maps, or even a tree-based analysis of these values in order to produce a multiscale characterization of land cover.

Finally, let us recall that extension of morphological operators to multivariate data is far from being trivial (while necessary if several observations or values are provided for each pixel). Two main strategies [8] are available: while the marginal strategy simply consists in a componentwise processing where each channel is treated independently from the others (as a greyscale image), the vectorial strategy is more theoretically sound. In this context, each pixel is assigned a vector, and these vectors are further compared through a vector ordering method. These extensions are also valid for some tree-based representations (inclusion trees) while some others (partitioning trees) generally require distance functions, that are usually easier to define in a multidimensional space.

\section{CONCLUSION}

Conversely to other satellite imaging modalities, microwave remote sensing is far from taking full advantage of recent advances in (spatial) image analysis. We have tackled this issue and describe how a very popular image analysis framework, namely Mathematical Morphology, could be used on SMOS data. Three different applications were provided to underline the possible use of morphological operators, for image/object simplification, detection, and description respectively.

The preliminary experiments have shown the potential of Mathematical Morphology (and more generally of digital image processing) for SMOS L1C data. Other image modalities are now to be considered, as well as the design of complete solutions for realistic applications in collaboration with microwave specialists.

\section{ACKNOWLEDGMENT}

François Cabot (CESBIO/CNES) for fruitful discussions on SMOS mission and microwave remote sensing.

\section{REFERENCES}

[1] P. Soille and M. Pesaresi, "Advances in mathematical morphology applied to geoscience and remote sensing," IEEE Transactions on Geoscience and Remote Sensing, vol. 40, no. 9, pp. 2042-2055, January 2002.

[2] P. Soille, Morphological Image Analysis : Principles and Applications, Springer-Verlag, Berlin, 2nd edition, 2003.

[3] P. Salembier and M. H. F. Wilkinson, "Connected operators," IEEE Signal Processing Magazine, vol. 26, no. 6, pp. 136-157, 2009.

[4] S. Peischl, J.P. Walker, C. Rüdiger, N. Ye, Y.H. Kerr, E. Kim, R. Bandara, and M. Allahmoradi, "The AACES field experiments: SMOS calibration and validation across the murrumbidgee river catchment," Hydrology and Earth System Sciences, vol. 16, pp. 1697-1708, 2012.

[5] P. Soille, "Constrained connectivity for hierarchical image partitioning and simplification," IEEE Transactions on Pattern Analysis and Machine Intelligence, vol. 30, no. 7, pp. 1132-1145, July 2008.

[6] M. Pesaresi and J. Benediktsson, "A new approach for the morphological segmentation of high resolution satellite imagery," IEEE Transactions on Geoscience and Remote Sensing, vol. 39, no. 2, pp. 309-320, February 2001.

[7] M. Dalla Mura, J. A. Benediktsson, B. Waske, and L. Bruzzone, "Morphological attribute profiles for the analysis of very high resolution images," IEEE Transactions on Geoscience and Remote Sensing, vol. 48, no. 10, pp. 3747-3762, 2010.

[8] E. Aptoula and S. Lefèvre, "A comparative study on multivariate mathematical morphology," Pattern Recognition, vol. 40, no. 11, pp. 2914-2929, November 2007. 